\section{Visión Electrónica Más que un estado sólido \\ https://doi.org/10.14483/issn.2248-4728}

UNIVERSIDAD DISTRITAL

FRANCISCO JOSÉ DE CALDAS

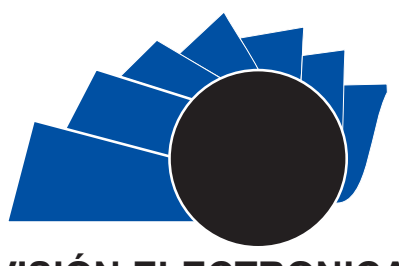

VISIÓN ELECTRONICA

Visión Actual

\title{
Pequeñas centrales hidroeléctricas $(\mathrm{PCH})$ : una revisión
}

\author{
Small hydropower plants (SHP): a review \\ Andrés Escobar Díaz ${ }^{1}$, Cristhian Leonardo Cortés Arenas ${ }^{2}$, Juan Sebastián Leguizamón Archila ${ }^{3}$
}

\section{INFORMACIÓN DEL ARTICULO}

Historia del articulo

Enviado: 18/12/2017

Recibido: 24/02/2018

Aceptado: 07/05/2018

\section{Palabras clave:}

Desarrollo sostenible,

Fuentes de energía renovable,

Generación de energía

hidroeléctrica,

Pequeña central hidroeléctrica,

Turbinas.

\section{Keywords:}

Sustainable development, Renewable energy sources, Hydroelectric power generation, Small hydro power plant, Turbines

\section{RESUMEN}

En el presente artículo se revisan los aspectos básicos a tener en cuenta dentro del planteamiento de un proyecto de Pequeñas Centrales Hidroeléctricas $(\mathrm{PCH})$, realizando énfasis en las metodologías de formulación y un acercamiento a los equipos electromecánicos vinculados al proceso de generación de energía. Se obtiene la descripción de cada fase de prefactibilidad de un proyecto de $\mathrm{PCH}$, así como los puntos clave y/o críticos asociados a su desarrollo y posible implementación en el marco de generación de energía colombiano. Adicionalmente, se abordan los conceptos básicos a considerar para el desarrollo de una propuesta de $\mathrm{PCH}$ y se hace énfasis en el trabajo de costos y diseño de turbinas como elementos primordiales para su implementación exitosa.

\begin{abstract}
:
The objective of this article is to review the basic aspects to be taken into account in the planning of a Small Hydroelectric Power Plants (SHP) project, emphasizing the formulation methodologies and an approach to the electromechanical equipment linked to the power generation process. Based on this, a description was made of each pre-feasibility phase of a SHP project, as well as the key and / or critical points associated with its development and possible implementation within the framework of Colombian energy generation. In addition, the basic concepts to be considered for the development of the proposal are addressed and emphasis is placed on the cost and design of turbines as key elements for its successful implementation.
\end{abstract}

1 Ingeniero Electrónico, Universidad Distrital Francisco José de Caldas, Colombia. MBA, Universidad de los Andes, Colombia. MSc. En Ingeniería Electrónica, Universidad de los Andes, Colombia. Docente Universidad Distrital Francisco José de Caldas, Colombia. Correo electrónico: aescobard@udistrital.edu.co. ORCID: https://orcid.org/0000-0003-0527-8776.

2 Tecnólogo en Electrónica, Universidad Distrital Francisco José de Caldas, Colombia. Ingeniería En Control C, Universidad Distrital Francisco José de Caldas, Colombia. Miembro Grupo de Investigación ORCA Universidad Distrital Francisco José de Caldas, Colombia. Trabajo actual: Pc Protección Car, Correo electrónico: cristhian.lcortes@gmail.com. ORCID: https://orcid.org/0000-0003-1342-1237.

3 Tecnólogo en Electrónica, Universidad Distrital Francisco José de Caldas, Colombia. Ingeniería En Control C, Universidad Distrital Francisco José de Caldas, Colombia. Miembro Grupo de Investigación ORCA Universidad Distrital Francisco José de Caldas, Colombia. Trabajo actual: Fundación Escuela Nueva. Correo electrónico: jsleguizamon@escuelanueva.org. ORCID: https://orcid.org/0000-0002-7698-1648

Citar este artículo como: A. Escobar-Díaz, C. L. Cortés-Arenas y J. S. Leguizamón-Archila, "Pequeñas centrales hidroeléctricas (PCH): Una revisión”, Visión electrónica, algo más que un estado sólido, vol. 1, no. 1, Edición especial, enero-junio 2018. DOI revista: https://doi.org/10.14483/issn.2248-4728. 


\section{Introducción}

Durante años, las crisis originadas por incrementos en los precios de los hidrocarburos y/o modelos energéticos tradicionales o comunes ha propiciado el desarrollo de recursos renovables autóctonos e inextinguibles, por encima de los elementos de tipo fósil (limitados en espacio y tiempo) [1-2].

En el marco de la idea anterior, el recurso a investigar, aprovechar y utilizar es el agua y su potencial hidrológico, a partir del concepto de "filo de agua". Este modelo consiste en la desviación de una parte del cauce del río para ser llevado a una planta de generación hidroenergética adecuada para tales condiciones de trabajo, [3].

Ante la escasa documentación, teniendo en cuenta la adecuación del modelo a las $\mathrm{PCH}$; el presente artículo establece un estado de conocimiento que sirva para constituir una metodología de formulación y un acercamiento a los equipos electromecánicos vinculados al proceso de generación de energía en una $\mathrm{PCH}$.

Por lo anterior, el desarrollo de cada capítulo de este documento pretende identificar puntos clave asociados al análisis, diseño y ejecución de este tipo de proyectos en base a las políticas y condiciones de operación para Colombia, un país con un $90 \%$ de potencial hidroenergético por aprovechar, [4].

El documento se estructura así: inicialmente se dan unas generalidades sobre estructura de $\mathrm{PCH}$; luego se indican los elementos prácticos para formular una $\mathrm{PCH}$; seguidamente sobre la viabilidad económica; luego elementos de diseño de las turbinas; posteriormente se describen algunos casos de éxito de PCH en Colombia; y finalmente se presentan las conclusiones de la investigación.

\section{Generalidades}

En este apartado se abordan los conceptos básicos aplicados al desarrollo de una Pequeña Central Hidroeléctrica vistos desde su implementación e incidencias dentro de un proyecto energético.

\subsection{Pequeñas Centrales Hidroeléctricas $(\mathbf{P C H})$}

Las PCH son centrales de generación de energía eléctrica con una capacidad instalada, dada en $\mathrm{kW}$, baja respecto a otros modelos productores, [5]. También pueden considerarse como un conjunto de obras civiles y equipos electromecánicos e hidráulicos cuyo objetivo es transformar la energía potencial y cinética del agua en movimiento en energía eléctrica útil para las necesidades asociadas a la zona de desarrollo de un proyecto energético, [6].

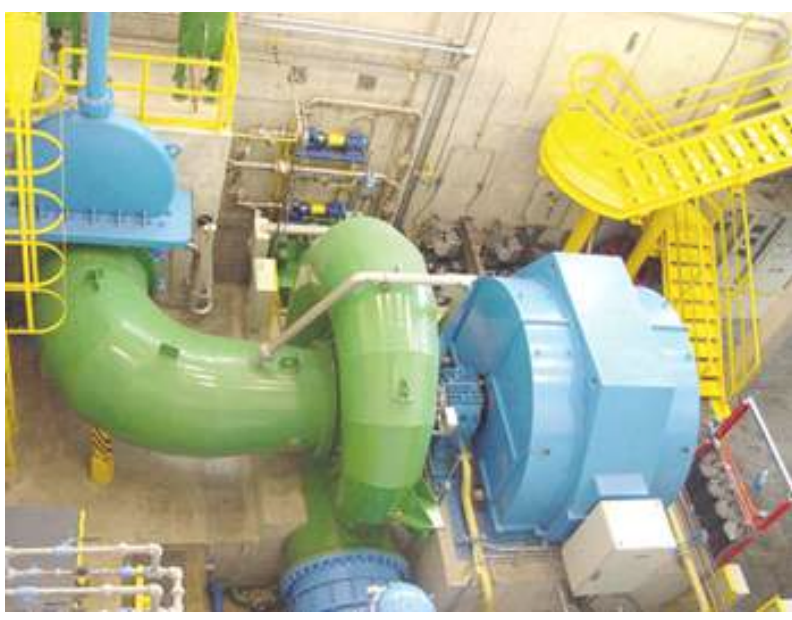

Figura 1. Pequeña Central Hidroeléctrica Santa Ana [7].

A partir de este concepto, las $\mathrm{PCH}$ se pueden dividir en cuatro tipos, considerando el rango de generación de energía que tengan en el momento de su implementación, así: en las Tablas 1 y 2, se observan los estándares establecidos por la Comisión de Regulación de Energía y Gas - CREG y por la Organización Latinoamericana de Energía OLADE, [8].

\begin{tabular}{|l|l|}
\hline \multicolumn{1}{|c|}{ Potencia } & \multicolumn{1}{c|}{ Tipo } \\
\hline $0-100 \mathrm{~kW}$ & Microcentral \\
\hline $100-1000 \mathrm{~kW}$ & Minicentral \\
\hline $1000-10000 \mathrm{~kW}$ & Pequeña Central \\
\hline
\end{tabular}

Tabla 1. Clasificación de PCHs, según la CREG [8].

\begin{tabular}{|c|l|c|c|c|}
\hline \multirow{2}{*}{ Potencia } & \multirow{2}{*}{ Tipo } & \multicolumn{3}{|c|}{ Salto } \\
\cline { 3 - 5 } & & Bajo & Medio & Alto \\
\hline $0.5-5 \mathrm{~kW}$ & Picocentral & \multicolumn{3}{|c|}{ N.A. } \\
\hline $5-50 \mathrm{~kW}$ & Microcentral & $<15$ & $15-50$ & $>50$ \\
\hline $50-500 \mathrm{~kW}$ & Minicentral & $<20$ & $20-100$ & $>100$ \\
\hline $500-5000 \mathrm{~kW}$ & Peq. Central & $<25$ & $25-130$ & $>130$ \\
\hline
\end{tabular}

Tabla 2. Clasificación de PCHs, según la OLADE [8].

\subsection{Partes de una $\mathrm{PCH}$}

Luego del concepto de Pequeña Central Hidroeléctrica; se evalúan las obras civiles básicas del proyecto para tener un marco general sobre las consideraciones requeridas a la hora de desarrollar un proceso de prefactibilidad de este tipo de plantas generadoras de energía. 


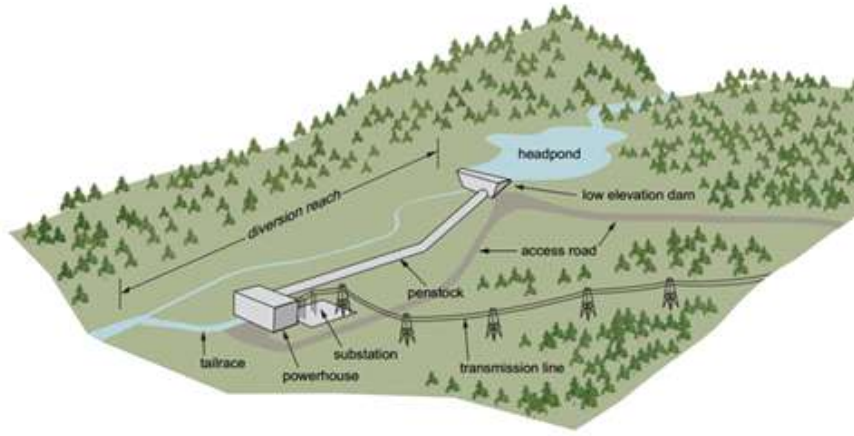

Figura 2. Estructura básica de una PCH tipo Filo de Agua [9].

\subsubsection{Captación o bocatoma}

Capta el agua para el funcionamiento de la $\mathrm{PCH},[10]$. Las partes principales de esta etapa son:

a. Barraje: es un dique transversal al río que debe levantar el tirante de agua en tiempo de estiaje y lograr que ingrese el agua al canal de demasías por la ventana de captación.

b. Muros de encauzamiento: son muros de concreto que encauzan las aguas del río, protegen la ventana de captación y sirven como defensa primaria del río.

c. Ventana de captación: permite el ingreso del agua del río al canal de demasías.

d. Canal de demasías: está asociado a los primeros metros del canal de conducción, pero maneja dimensiones mayores garanticen la conducción de mayor cantidad de agua sin ocasionar desbordes.

\subsubsection{Canal}

Conduce el agua desde la toma o bocatoma hasta la cámara de carga, se caracteriza por ser de tipo abierto y por tener una unión de material hidrófugo que impide la pérdida de agua por percolación, y mejor acabado para mitigación de efectos por fricción, [10].

\subsubsection{Aliviadero o vertedero}

Permite la eliminación, de manera segura, del exceso de caudal de agua producto de las épocas de lluvia presentes en la zona de ubicación del proyecto, [10].

\subsubsection{Desarenador}

Sedimenta partículas de tierra y arena que son arrastradas a lo largo del canal, y evita su ingreso al sistema de tuberías de la planta, [10].

\subsubsection{Cámara de carga}

Es un depósito situado al final del canal y antes de la entrada de la tubería de fuerza que permite mantener una reserva de agua en caso de pérdida de presión en la misma; también es utilizado como último filtro para limpieza del agua de sedimentos que no sean retirados en el desarenador, [11].

\subsubsection{Tubería de presión}

Conduce el agua a presión desde la cámara de carga hacia la turbina, el propósito de que sea forzada es mantener las condiciones de entrada durante la transferencia. Se debe definir el tipo de material para saber que partes deberá llevar la instalación y los acabados requeridos, [11].

\subsubsection{Casa de máquinas}

Protege, ubica y cimienta adecuadamente el equipo electromecánico además de almacenar repuestos y equipos propios de la planta de generación de energía. En caso de no ser totalmente automatizada, la PCH contará con personal en esta área encargada de supervisión, control y revisión del proceso de funcionamiento óptimo [10].

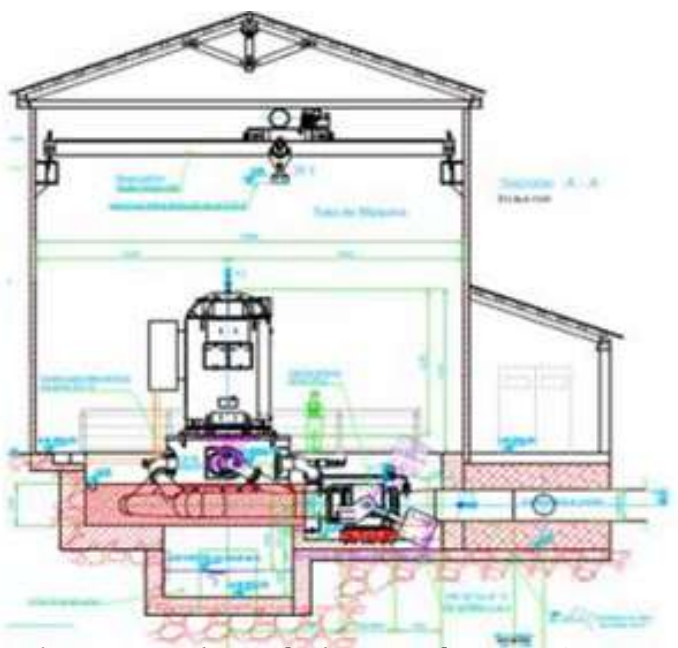

Figura 3. Plano de la casa de máquinas de una $\mathrm{PCH}[12]$.

\subsubsection{Canal de descarga}

Permite el retorno del agua hacia el río una vez se ha generado el movimiento sobre las turbinas de impulsión. Cabe resaltar que los diseños de estos canales deben garantizar la ausencia de erosión en los cimientos de la $\mathrm{PCH}$ a causa de la fuerza de salida del líquido, [10]. 


\section{Metodología de formulación de una PCH}

Para llegar a un punto de formulación y posterior autorización y/o ejecución de una $\mathrm{PCH}$, se hace vital realizar un proceso de investigación que revele las condiciones reales de trabajo y las alternativas de solución para el desarrollo del proyecto desde el punto de vista tecno-económico.

Bajo este razonamiento, se desplegarán dos (2) propuestas, basadas en casos ampliamente documentados, que permiten identificar el paso a paso y las condiciones de trabajo óptimas para su ejecución.

\subsection{Criterios básicos de implementación-Caso I}

En base a la documentación obtenida, se describirá la primera propuesta-Figura 4-con cada punto de ejecución, sus implicaciones y los requerimientos para la concepción de la $\mathrm{PCH}$

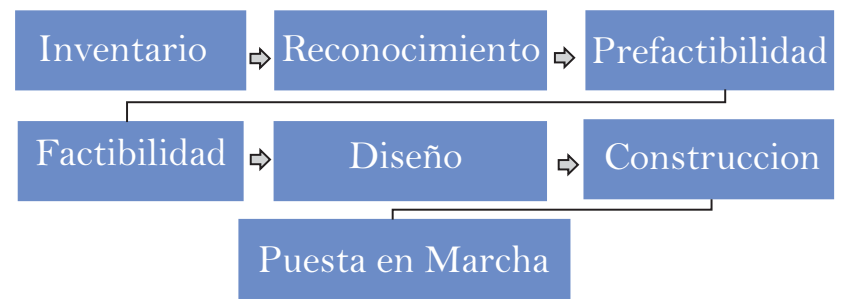

Figura 4. Propuesta para desarrollo de proyecto de PCH. Fuente: Elaboración propia.

\subsubsection{Inventario}

Esta primera fase radica en un estudio que permite identificar condiciones básicas aplicables al desarrollo de una PCH, [13]. Se divide en dos estudios particulares:

a) Estudio de poblaciones

Se busca información sobre grupos que no tengan acceso, indistintamente de las causas, al servicio de energía eléctrica para determinar las circunstancias de demanda e instalación de PCH.

b) Estudio de recursos hidroenergético

Se estiman las posibilidades del recurso a manejar a partir de estudios topográficos, geológicos e hidrológicos para que el proyecto sea ejecutado de acuerdo a estas condiciones particulares, [14].

\subsubsection{Reconocimiento}

Debido a que, en algunos casos, la información obtenida en la etapa de inventario no permite identificar de manera completa la situación real de la población, y de las condiciones que rodean la implementación de una $\mathrm{PCH}$, se hace necesaria una visita de reconocimiento al sector que se está considerando para la ejecución del proyecto buscando obtener toda la información vinculada a estudios de poblaciones y de recursos hidroenergéticos. De esta fase se deben generar evidencias a escala que garanticen los datos de viabilidad críticos para la ejecución de las obras que requiere el proyecto, [6].

\subsubsection{Prefactibilidad}

Esta etapa es crítica porque recoge toda la información obtenida en las fases previas, y se condensa al punto de reducir los posibles lugares para la implementación de $\mathrm{PCH}$, mitigando los efectos negativos -generalmente medioambientales- que siempre han estado vinculados a este tipo de proyectos. Para poder realizar un correcto análisis de prefactibilidad se debe considerar un predimensionamiento de las obras y un estimado de los costos generados tales que permitan tomar una decisión frente a: continuar, modificar o terminar las investigaciones asociadas a la $\mathrm{PCH}$ en un punto particular preseleccionado durante la fase de inventario o la de reconocimiento [15], [16]. Bajo esta premisa los puntos clave a evaluar son:

a) Localización de los sitios de obra: ubicación de las obras de derivación del río, estrechamiento del cauce del río, desviación del río, estabilidad de las zonas de construcción de las obras y daños asociados a condiciones del terreno.

b) Información básica: pendiente y perfil transversal del río, cotas en los sitios de las obras con derivación, longitud de conducción y perfil preliminar de la tubería, [13].

\subsubsection{Factibilidad}

En este punto se realiza el último estudio sobre las condiciones de operación que estarán presentes en el diseño final de la PCH. En sí, el proceso es similar a su fase previa pero su grado de incertidumbre se reduce de tal forma que el proyecto sea ejecutable y sus posibles pérdidas sean mínimas [17]. Los puntos críticos a evaluar dentro de esta fase se describen a continuación:

a) Estimación de la demanda: en este análisis se verifica el horizonte de planeación, el número de 
habitantes beneficiados con este futuro servicio, la magnitud de los sectores de demanda a partir de su carga residencial, de alumbrado público, comercial e industrial, la relación hora vs potencia, consumo de los diferentes equipos electrónicos, la curva de demanda de un día representativo del año, la mayor carga pico, la potencia media, y el factor de carga.

b) Encuestas: a pesar de no ser un elemento destacado dentro del desarrollo de una $\mathrm{PCH}$, es vital, a la hora de ofrecer un servicio a futuros consumidores, conocer las condiciones del mercado y la manera correcta de acceder a las poblaciones que requieran la energía que se pretende comercializar,[18]. Dado esto, se pueden tener en cuenta algunos elementos a la hora de solicitar información de las poblaciones afectadas o beneficiadas por la presencia de una PCH en el área circundante como:

- Marco general: Nombre del pueblo, ubicación, características del pueblo, vías de acceso, medios de transporte, tipo de comunidad, líderes de la comunidad, organizaciones comunitarias de industriales, migración, emigración, natalidad, mortalidad, y fuentes de energía usadas por la comunidad.

- Actividades residenciales: Número de viviendas, características de las viviendas, número de habitantes por vivienda, actividades comunes y el uso energético en esas actividades.

- Actividades industriales y comerciales: Tipo de actividad, ingresos y uso de energéticos.

- Servicios públicos: Acueducto y alcantarillado, salud pública, telecomunicaciones, energía eléctrica, alumbrado público, educación y entidades oficiales.

c) Potencia: finalmente, se necesita conocer el consumo energético que puede generar la población vinculada al proyecto para verificar que la producción pueda responder a estas necesidades sin tener pérdidas de tipo económico o energético, de ser el caso. Este estudio se realiza entre la potencia firme y la demanda del sistema, obteniendo evidencias claras que permitan decidir sobre la reducción de la producción de la planta o su reestructuración para acondicionarse a la comunidad vinculada al proyecto, [13].

\subsubsection{Diseño}

El diseño final se inicia una vez se tiene claridad frente a la propuesta y el lugar de implementación, partiendo de todos los estudios que se han podido observar en las fases previas descritas. Aquí se realiza el dimensionamiento de cada pieza, la selección de los instrumentos de medición y automatización, y la adecuación general de la planta considerando todas las exigencias obtenidas en los estudios desarrollados, [19]. De este modo, en esta fase se calcula:

- Cantidades de obra de todos los rubros y componentes a emplear.

- Precios unitarios, mano de obra, adquisición y transporte de materiales, alquiler de maquinaria y demás que se vinculen a un valor cuantitativo.

- Presupuesto total.

\subsection{Criterios básicos de implementación-Caso 2}

Para este segundo caso, se observarán las consideraciones requeridas para poder llevar a cabo la puesta en funcionamiento de una $\mathrm{PCH}$, figura 5 .

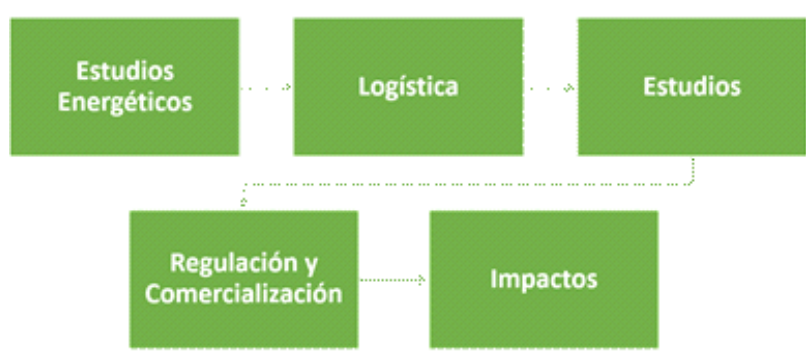

Figura 5. Propuesta para desarrollo de proyecto de PCH. Fuente: Elaboración propia.

\subsubsection{Estudios energéticos}

Se realizan a partir de un análisis de las condiciones históricas y actuales del mercado para toma de decisiones, [4].

- Negociación y opciones de venta

Se busca proyectar la inversión necesaria para cubrir las necesidades evaluadas en el punto previo y, de este modo, negociar el recurso con el Ministerio de Minas y Energía (MinMinas), la bolsa de energía, los usuarios finales y futuros, [20].

\subsubsection{Logística}

Hace referencia a los elementos necesarios antes, durante y después del proyecto para lograr su correcto desarrollo y revisión por personal de la planta, entes de 
control, inversionistas, entre otros, [4].

- Vías de acceso.

- Conexión eléctrica.

\subsubsection{Estudios de área de influencia}

El Estudio de Impacto Ambiental (EIA) debe delimitar y definir las áreas de influencia del proyecto con base en una identificación de los impactos que puedan generarse durante su construcción y operación. Para los medios abióticos y bióticos, se tendrán en cuenta unidades fisiográficas, naturales y ecosistémicas; y para los aspectos sociales, las entidades territoriales y las áreas étnicas de uso social, económico y cultural entre otros, asociadas a las comunidades asentadas en dichos territorios, $[21]$.

De este modo, los estudios se realizan desde dos puntos de vista, según la influencia del proyecto, así:

a) Área de influencia directa (AID): es aquella donde se manifiestan los impactos generados por las actividades de construcción y operación; está relacionada con el sitio del proyecto y su infraestructura, [22].

b) Área de influencia indirecta (AII): es aquella donde los impactos trascienden el espacio físico del proyecto y su infraestructura asociada, [23].

El proyecto, a partir de estas consideraciones, deberá dar la siguiente información (Tabla 3):

\begin{tabular}{|c|c|c|}
\hline $\begin{array}{l}\text { Tipo de } \\
\text { estudio }\end{array}$ & Área de Influencia Directa (AID) & Área de Influencia Indirecta (AII) \\
\hline Geología & $\begin{array}{l}\text { Presentar la cartografía geológica detallada } \\
\text { (unidades y rasgos estructurales) y } \\
\text { actualizada con base en fotointerpretación y } \\
\text { control de campo. }\end{array}$ & $\begin{array}{l}\text { Describir las unidades litológicas y rasgos } \\
\text { estructurales, con base en estudios } \\
\text { existentes en la zona y ajustadas con } \\
\text { información de sensores remotos y control } \\
\text { decampo. } \\
\text { Identificar y localizar las amenazas } \\
\text { naturales como movimientos en masa, } \\
\text { sismicidad y fallas geológicas activas. }\end{array}$ \\
\hline Geomorfología & \multicolumn{2}{|c|}{$\begin{array}{l}\text { Definir las unidades geomorfológicas a partir del análisis de morfogénesis, } \\
\text { morfografía, morfodinámica y morfoestructuras. }\end{array}$} \\
\hline Suelos & $\begin{array}{l}\text { Realizar la clasificación de los suelos, con base } \\
\text { en información primaria y secundaria } \\
\text { considerando el ordenamiento territorial } \\
\text { municipal. }\end{array}$ & $\begin{array}{l}\text { Realizar la clasificación de los suelos, con } \\
\text { base en la interpretación de información } \\
\text { secundaria y considerando el ordenamiento } \\
\text { territorial municipal. }\end{array}$ \\
\hline Hidrología & $\begin{array}{l}\text { Identificar el tipo y distribución de las redes } \\
\text { de drenaje, describir y localizar la red } \\
\text { hidrográfica e identificar la dinámica fluvial } \\
\text { de las fuentes que pueden ser afectadas por el } \\
\text { proyecto, así como las posibles alteraciones } \\
\text { de su régimen natural. } \\
\text { Realizar el inventario de las principales } \\
\text { fuentes contaminantes, identificando el } \\
\text { generador y tipo de vertimiento y determinar } \\
\text { el régimen hidrológico y los caudales } \\
\text { máximos, medios, mínimos mensuales y } \\
\text { multianuales delas fuentes a intervenir. }\end{array}$ & $\begin{array}{l}\text { Identificar los sistemas lénticos y lóticos, } \\
\text { establecer los patrones de drenaje a nivel } \\
\text { regional e identificar el régimen } \\
\text { hidrológico y de caudales característicos de } \\
\text { las principales corrientes. }\end{array}$ \\
\hline
\end{tabular}




\begin{tabular}{|c|c|c|}
\hline $\begin{array}{l}\text { Calidad del } \\
\text { Agua }\end{array}$ & $\begin{array}{l}\text { Para las fuentes de agua susceptibles de } \\
\text { intervención, realizar la caracterización físico- } \\
\text { química, bacteriológica e hidrobiológica, } \\
\text { considerando al menos dos periodos climáticos } \\
\text { (época s eca y é p oc a de lluvias ). }\end{array}$ & No Aplica. \\
\hline Uso del Agua & $\begin{array}{l}\text { Determinar los posibles conflictos actuales o } \\
\text { potenciales sobre la disponibilidad y usos del } \\
\text { agua, teniendo en cuenta el análisis de } \\
\text { frecuencias de caudales mínimos para } \\
\text { diferentes períodos de retorno. }\end{array}$ & No Aplica. \\
\hline Hidrogeología & $\begin{array}{l}\text { Realizar el inventario de puntos de agua, } \\
\text { establecer las unidades hidrogeológicas que } \\
\text { intervendrá el proyecto y evaluar la } \\
\text { vulnerabilidad a la contaminación de las aguas } \\
\text { subterráneas y zonas de recarga y descarga. }\end{array}$ & $\begin{array}{l}\text { Identificar el tipo de acuífero, establecer las } \\
\text { direcciones de flujo e identificar las zonas } \\
\text { de recarga y descarga. }\end{array}$ \\
\hline Geotecnia & \multicolumn{2}{|c|}{$\begin{array}{l}\text { Con base en la información geológica, edafológica, geomorfológica, } \\
\text { hidrogeológica, hidrológica, climatológica y de amenaza sísmica, realizar la } \\
\text { zonificación y cartografía geotécnica }\end{array}$} \\
\hline Atmósfera & \multicolumn{2}{|c|}{$\begin{array}{l}\text { Clima: Identificar, zonificar y describir las condiciones climáticas mensuales } \\
\text { multianuales del área. } \\
\text { Calidad del aire: Evaluar su calidad considerando las fuentes de emisiones atmosféricas } \\
\text { existentes en la zona y la ubicación de los asentamientos poblacionales. } \\
\text { Ruido: Considerar las fuentes de generación existentes en la zona y la ubicación de los } \\
\text { asentamientos poblacionales. }\end{array}$} \\
\hline Paisajes & \multicolumn{2}{|c|}{$\begin{array}{l}\text { Utilizar sensores remotos como imágenes de satélite, radar o fotografías aéreas para } \\
\text { establecer las unidades de paisaje regional y su interacción con el proyecto }\end{array}$} \\
\hline $\begin{array}{l}\text { Ecosistemas } \\
\text { Terrestres }\end{array}$ & $\begin{array}{l}\text { Para flora: localizar, caracterizar y cuantificar } \\
\text { las diferentes unidades de cobertura vegetal, el } \\
\text { uso actual del suelo, las diferentes unidades } \\
\text { florísticas, los usos dados por la comunidad a } \\
\text { estas especies y la biomasa vegetal que será } \\
\text { afectada por el proyecto. } \\
\text { Para fauna: determinar las principales } \\
\text { cadenas tróficas, fuentes naturales de } \\
\text { alimentación y rutas migratorias de las } \\
\text { especies, la dinámica de la fauna silvestre, los } \\
\text { estados poblacionales de las especies } \\
\text { reconocidas y aspectos de densidad y } \\
\text { diversidad de las especies con condiciones } \\
\text { particulares de amenaza. }\end{array}$ & $\begin{array}{l}\text { Para flora: identificar, sectorizar y } \\
\text { describir las zonas de vida o formaciones } \\
\text { vegetales, los diferentes tipos de cobertura } \\
\text { vegetal y los ecosistemas sensibles y áreas } \\
\text { naturales protegidas. } \\
\text { Para fauna: identificar la fauna asociada a } \\
\text { las diferentes unidades de coberturas } \\
\text { ve g e tal e s e n b a s e a e s peci e s } \\
\text { representativas, de valor comercial, } \\
\text { endémicas, amenazadas o en peligrocrítico. }\end{array}$ \\
\hline $\begin{array}{l}\text { Ecosistemas } \\
\text { Acuáticos }\end{array}$ & $\begin{array}{l}\text { Caracterizar los ecosistemas con base en el } \\
\text { levantamiento de información primaria, } \\
\text { analizar sus diferentes hábitats, su distribución } \\
\text { espacial y temporal y las interrelaciones con } \\
\text { otros ecosistemas y subsistemas. }\end{array}$ & $\begin{array}{l}\text { Identificar los principales ecosistemas y } \\
\text { determinar su dinámica e importancia en el } \\
\text { contexto regional y la biota asociada a los } \\
\text { cuerpos de agua de mayor importancia a } \\
\text { nivel e cológ i c o y e conómico. }\end{array}$ \\
\hline
\end{tabular}




\begin{tabular}{|c|c|c|}
\hline $\begin{array}{l}\text { Lineamientos de } \\
\text { Participación }\end{array}$ & $\begin{array}{l}\text { Informar y comunicar los alcances del proyecto } \\
\text { y sus implicaciones de todo tipo con sus } \\
\text { respectivas medidas de mitigación a los } \\
\text { ciudadanos, comunidades organizadas y } \\
\text { comunidades étnicas. }\end{array}$ & $\begin{array}{l}\text { Acercar e informar sobre el proyecto y sus } \\
\text { implicaciones a las autoridades regionales.. }\end{array}$ \\
\hline $\begin{array}{l}\text { Dimensión } \\
\text { Demográfica }\end{array}$ & $\begin{array}{l}\text { Caracterizar los grupos poblaciones y la } \\
\text { dinámica poblacional a partir de las unidades } \\
\text { territoriales afectadas por el proyecto. }\end{array}$ & $\begin{array}{l}\text { Analizar la dinámica de poblamiento } \\
\text { histórica, actual y futura además del tipo de } \\
\text { población a sentada. }\end{array}$ \\
\hline $\begin{array}{l}\text { Dimensión } \\
\text { Espacial }\end{array}$ & $\begin{array}{l}\text { Hacer un análisis de calidad, cobertura, } \\
\text { infraestructura asociada, debilidad y } \\
\text { potencialidades de los servicios públicos, } \\
\text { sociales, de comunicación y de transporte. }\end{array}$ & $\begin{array}{l}\text { Hacer una síntesis regional de los servicios } \\
\text { públicos y sociales. }\end{array}$ \\
\hline $\begin{array}{l}\text { Dimensión } \\
\text { Económica }\end{array}$ & $\begin{array}{l}\text { Determinar las relaciones económicas, la } \\
\text { estructura, dimensión y distribución de la } \\
\text { producción y las dinámicas económicas locales, } \\
\text { para precisar en fases posteriores las variables } \\
\text { que se verán afectadas con las actuaciones del } \\
\text { p r o y e c t o. }\end{array}$ & $\begin{array}{l}\text { Identificar y analizar los procesos } \\
\text { existentes en la región en base a la } \\
\text { estructura de la propiedad, los procesos } \\
\text { productivos y tecnológicos y el mercado } \\
\text { laboral a ctual. }\end{array}$ \\
\hline $\begin{array}{l}\text { Dimensión } \\
\text { Cultural }\end{array}$ & $\begin{array}{l}\text { Estudiar los aspectos que puedan llegar a } \\
\text { afectar el modo de vida de las comunidades } \\
\text { étnicas o no étnicas en base a la dinámica de } \\
\text { poblamiento, los territorios, la etnolingüística, } \\
\text { la demografía, la salud, la educación, la } \\
\text { religiosidad, la economía tradicional, la } \\
\text { organización sociocultural y la presencia } \\
\text { institucional. }\end{array}$ & $\begin{array}{l}\text { Caracterización cultural de comunidades no } \\
\text { étnicas y étnicas a partir de hechos } \\
\text { históricos relevantes, economía tradicional, } \\
\text { organización social y apropiación de los } \\
\text { recursos naturales. }\end{array}$ \\
\hline $\begin{array}{c}\text { Aspectos } \\
\text { Arqueológicos }\end{array}$ & \multicolumn{2}{|c|}{$\begin{array}{l}\text { Desarrollar un proyecto de arqueología preventiva que permita visualizar el cumplimiento } \\
\text { de toda la normatividad en pro de solicitar las licencias ambientales. Se realiza desde los } \\
\text { puntos de diagnóstico y evaluación hasta el plan de manejo arqueológico requerido. }\end{array}$} \\
\hline
\end{tabular}

Tabla 3. Estudios para formulación de proyectos con base en sus áreas de influencia [21].

\subsubsection{Regulación y comercialización}

Se evalúan los factores que afectan el precio de la energía en el mercado para realizar estrategias de negocio a distintos tiempos, [24] figura 6 .

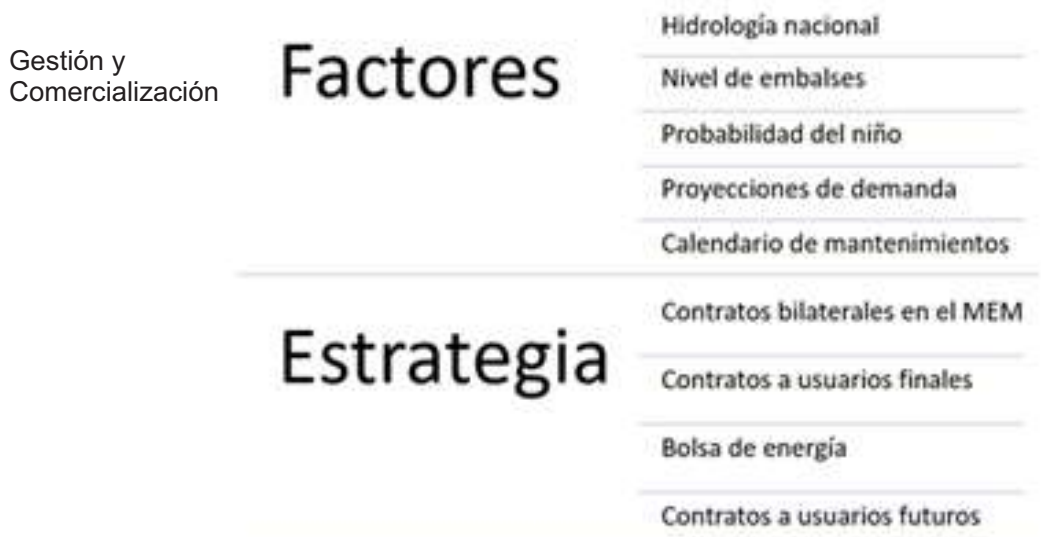

Figura 6. Gestión y comercialización de energía. Fuente: Elaboración propia. 
a) Tramitología para conexión de $\mathrm{PCH}$. Una vez se han verificado todos los factores que vinculen el correcto desarrollo de una $\mathrm{PCH}$, se transita hacia una fase de decisión que permita realizar la inscripción, licencias, estudios, financiación y construcción del proyecto, [20].

b) Conexión y puesta en operación. Para esta fase del proceso se requiere realizar un estudio de conexión con dos alternativas que garanticen un desempeño adecuado del proyecto y estén acordes con las políticas que rigen los mercados mayoristas y minoristas del sector energético, [25].

\subsubsection{Impactos}

Para cerrar este análisis, se deben verificar los impactos finales respecto a los identificados en la fase de estudios de áreas de influencia y garantizar que los procesos de mitigación sean constantes durante el desarrollo y puesta en operación de la planta.

a) Condiciones mínimas del proyecto. Los elementos mínimos a considerar, buscando mitigar efectos de impacto negativo en las áreas identificadas en la sección de estudios, se observan en la figura $7,[26]$.

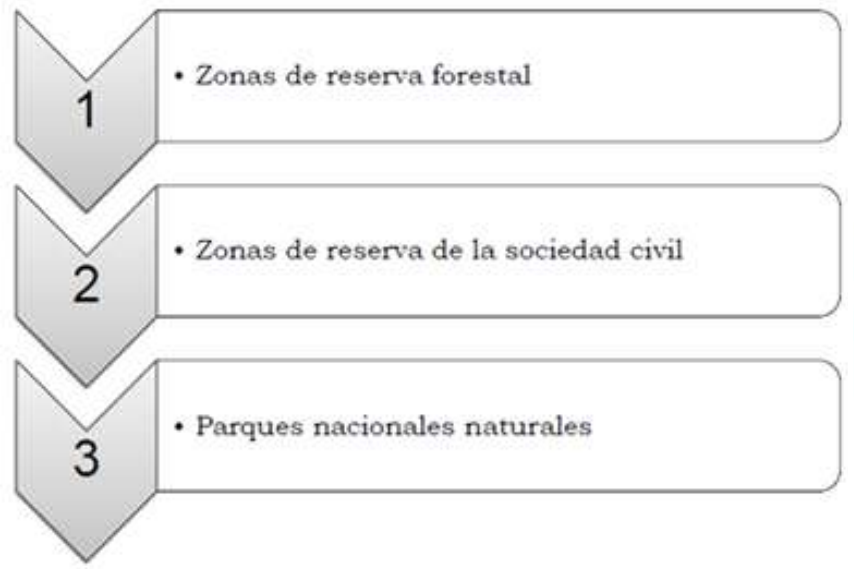

Figura 7. Tips para la toma de decisiones sobre ejecución de proyectos hidroenergéticos [26].

b) Determinación de áreas de influencia. Se deben definir las áreas de influencia directa e indirecta (AID y AII) para justificar las consultas previas y las condiciones de las licencias, [27].

c) Compensaciones forestales. A partir del manual de compensación forestal del Ministerio de Ambiente y Desarrollo Sostenible, tomar decisiones para la sustracción de áreas de reserva y permisos de aprovechamiento forestal [28].

\section{Análisis de costos}

A partir de diferentes casos reales de Pequeñas Centrales Hidroeléctricas, se realizan los diferentes análisis de costos buscando identificar puntos de mayor inversión, fuentes de riesgos para este tipo de proyecto, y las proyecciones producto del constante cambio de los mercados y la competencia con las tecnologías basadas en hidrocarburos y Grandes Centrales Hidroeléctricas. Se plantean para el efecto dos casos de estudio, ambos con los indicadores de inversión: en equipamiento, estructura, y administración. Tablas 4 y 5 ; figuras 8 y 9 .

\subsection{Caso I- PCH Portugal}

\begin{tabular}{|l|r|r|}
\hline $\begin{array}{l}\text { INVERSIONES } \\
\text { INICIALES }\end{array}$ & \multicolumn{1}{|c|}{ IMPORTE } & PORCENTAJE \\
\hline Estructura física & $1.884 .000,00 €$ & $42 \%$ \\
\hline $\begin{array}{l}\text { Inversión } \\
\text { administrativa }\end{array}$ & $298.600,00 €$ & $7 \%$ \\
\hline $\begin{array}{l}\text { Inversión de } \\
\text { equipos }\end{array}$ & $2.269 .000,00 €$ & $51 \%$ \\
\hline Total & $4.451 .600,00 €$ & $100 \%$ \\
\hline
\end{tabular}

Tabla 4. Inversión inicial del proyecto [29].

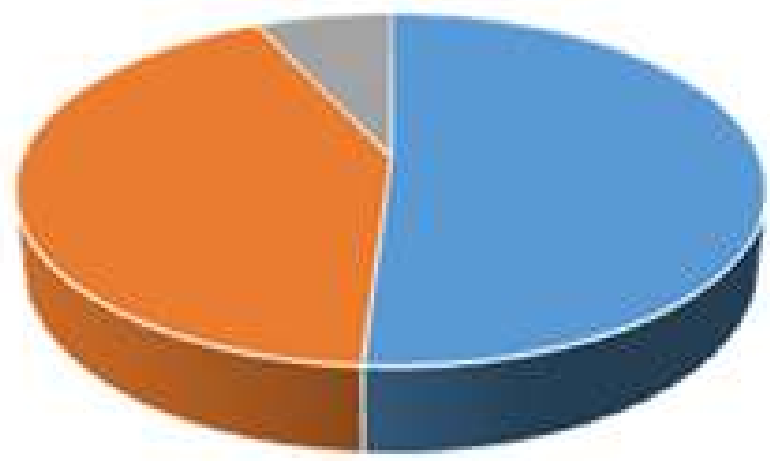

Figura 8. Inversión inicial de proyecto. Fuente: Elaboración propia. 


\subsection{Caso II - PCH}

\begin{tabular}{|l|c|r|}
\hline $\begin{array}{c}\text { INVERSIONES } \\
\text { INICIALES }\end{array}$ & IMPORTE & PORCENTAJE \\
\hline $\begin{array}{l}\text { Costos de } \\
\text { planificación y } \\
\text { diseño }\end{array}$ & USD 40.553,06 & $13 \%$ \\
\hline Trabajos civiles & USD $97.725,02$ & $32 \%$ \\
\hline $\begin{array}{l}\text { Equipos } \\
\text { electromecánicos }\end{array}$ & USD $171.198,49$ & $55 \%$ \\
\hline \multicolumn{1}{|c|}{ Total } & USD $\mathbf{3 0 9 . 4 7 6 , 5 7}$ & $\mathbf{1 0 0 \%}$ \\
\hline
\end{tabular}

Tabla 5. Inversión inicial del proyecto [30].

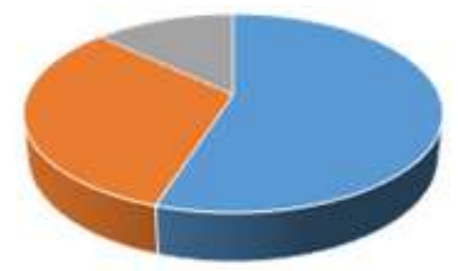

- Electro-mechanical equipment

- Civil works

= Planning and design costs

Figura 9. Inversión inicial del proyecto. Fuente: Elaboración propia.

\section{Turbinas}

Existen muchos tipos de turbinas aplicables a sistemas de energía hidroeléctrica. A menudo se investiga sobre el mejor tipo de estas partiendo de sus condiciones físicas; pero la manera adecuada de hacerlo es respecto a la más apropiada para el diseño que se tenga en consideración, [31]. De manera simple, se verán los diferentes tipos de turbina y se adopta un enfoque centrado en los modelos más utilizados en la implementación de PCH, tabla 6.

\begin{tabular}{|c|c|}
\hline $\begin{array}{l}\text { TIPO DE TURBINA } \\
\text { HIDROELÉCTRICA }\end{array}$ & CARACTERÍSTICAS TÍPICAS \\
\hline Tornillo arquimediano & $\begin{array}{l}\text { Cabezales bajos ( } 1,5-5 \text { metros) Caudales medianos a altos }\left(1 \text { a } 20 \mathrm{~m}^{3} /\right. \\
\text { s). Para flujos más altos se utilizan múltiples tornillos [32]. }\end{array}$ \\
\hline $\begin{array}{l}\text { Turbina de flujo } \\
\text { cruzado }\end{array}$ & $\begin{array}{l}\text { Cabezales bajos a medios }(2-40 \text { metros }) \\
\text { Caudales bajos a medios }\left(0,1 \_5 \mathrm{~m}^{3} / \mathrm{s}\right)[33] \text {. }\end{array}$ \\
\hline Turbina Kaplan & $\begin{array}{l}\text { Cabezales bajos a medios (1,5-20 metros) } \\
\text { Flujos medios a altos }\left(3 \mathrm{~m}^{3} / \mathrm{s}-30 \mathrm{~m}^{3} / \mathrm{s}\right) \text { Para flujos más altos, se pueden } \\
\text { usar múltiples turbinas. }\end{array}$ \\
\hline $\begin{array}{l}\text { Turbina Pelton / } \\
\text { Turgo }\end{array}$ & $\begin{array}{l}\text { Cabezas altas (más de } 25 \text { metros) } \\
\text { Caudales inferiores }\left(0,01 \mathrm{~m}^{3} / \mathrm{s}-0,5 \mathrm{~m}^{3} / \mathrm{s}\right)[34]\end{array}$ \\
\hline Ruedas de agua & $\begin{array}{l}\text { Cabezales bajos ( } 1-5 \text { metros }) \text {, aunque las turbinas a menudo son } \\
\text { más apropiadas para cabezales más altos. } \\
\text { Flujos medios }\left(0.3-1.5 \mathrm{~m}^{3} / \mathrm{s}\right) \text {. }\end{array}$ \\
\hline Turbina Francis & $\begin{array}{l}\text { Ya no se usa comúnmente, excepto en sistemas hidroeléctricos de } \\
\text { almacenamiento muy grandes, aunque existen muchas turbinas más } \\
\text { antiguas y más pequeñas que se pueden restaurar. } \\
\text { Para turbinas más antiguas: cabezas bajas a medianas ( } 1.5-20 \text { metros) } \\
\text { Flujos medios }(0.5-4 \mathrm{~m} 3 / \mathrm{s}) \text {. }\end{array}$ \\
\hline
\end{tabular}

Tabla 6. Tipos de turbinas hidroeléctricas [31].

\subsection{Turbina Kaplan}

Está diseñada para aplicaciones de baja caída de agua. La turbina Kaplan tiene las hélices como hojas, pero trabaja de manera inversa; esto quiere decir que en lugar de desplazar el agua axialmente utilizando la potencia del eje y la creación de empuje axial, la fuerza axial de agua actúa sobre las palas de la turbina y la generación de potencia en el eje, [35], [36].

Las condiciones iniciales a considerar para su diseño son: 
a) Se requieren grandes niveles de caudalatravesando la turbina para realizar la correcta generación de energía.

b) Más allá del diseño, su capacidad de producción de energía está entre $2 \mathrm{MW}$ y $100 \mathrm{MW}$.

c) Flujo de tipo radial.

\subsection{Turbina Francis}

El flujo de agua de la turbina es radial y sale axialmente. La presión del agua disminuye a medida que pasa a través de la reacción de impartición de la turbina en sus álabes que la hace girar, [37].

La turbina Francis es la primera turbina hidráulica con flujo de entrada radial. Fue diseñada por el científico estadounidense James Francis. Las turbinas de reacción tienen algunas características primarias que las diferencian de las turbinas de impulso: la mayor parte de la caída de presión se produce en la propia turbina, a diferencia de la turbina de impulso donde la caída de presión completa tiene lugar hasta el punto de entrada y el paso de la turbina se llena completamente por el flujo de agua durante la operación [38].

Las condiciones iniciales a considerar para su diseño son:

- La fuerza no depende estrictamente del caudal adquirido durante el recorrido del agua.

- Presenta un corredor que permite el flujo del líquido por toda la estructura de la turbina.

- El líquido presenta una pérdida de presión para incrementar y/o mantener la velocidad.

\subsection{Turbina Pelton}

En una turbina Pelton o Pelton Wheel, chorros de agua impactan en sus palas haciendo girar el volante, produciéndose el par y la consecuente potencia, [39].

La Pelton tiene un disco circular montado sobre el eje giratorio o rotor. Este disco circular tiene forma de copa cuchillas, llamados cubos, colocados a igual separación alrededor de su circunferencia. Las boquillas están dispuestas alrededor de la rueda de tal manera que el chorro de agua que emerge de una boquilla es tangencial a la circunferencia de la rueda de Turbina Pelton. De acuerdo con la carga disponible de agua (presión de agua), y los requisitos de funcionamiento de la forma, el número de boquillas alrededor de la rueda Pelton puede variar, [40].

\subsection{Rangos de utilización y eficiencia}

En PCH, el desarrollo de sistemas de automatización y control y la estandarización de los equipos electromecánicos, basados en investigación y desarrollo tecnológico, permiten que los esquemas desarrollados adquieran un fundamento sólido y adaptable a los ambientes y situaciones de aplicación, [41].

Con base en la anterior afirmación, el diseño e implementación de las turbinas, deben ser enfocados en las dos condiciones más importantes vinculadas al fluido y la generación de energía: la altura de salto (grande o pequeño), y el caudal del fluido (variable o constante, alto o bajo).

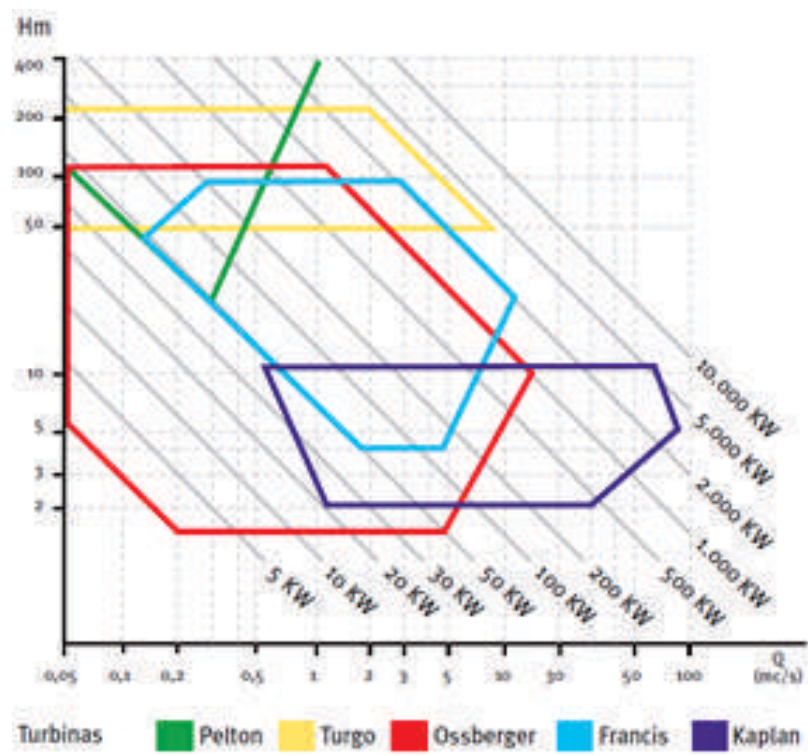

Figura 10. Campo de utilización de los diferentes tipos de turbinas para PCHs [11]

Verificando la figura 10, que evidencia el rango de utilización de las principales turbinas para aplicaciones de tipo hidroeléctrico, se pueden dar tres consideraciones principales para una selección inicial dentro de un proyecto de $\mathrm{PCH}$ :

- Turbina Kaplan: Saltos pequeños y caudales variables.

- Turbina Francis: Saltos más elevados y variaciones de caudal moderadas.

- Turbina Pelton: Grandes salto, independientes de la variación del caudal.

Con este acercamiento previo y algunas características adicionales de cada tipo de turbina como velocidad específica, tamaño del rodete, velocidad de rotación síncrona y otros elementos constitutivos de cada una se puede hallar el equipo electromecánico óptimo para el proyecto de $\mathrm{PCH}$ que se requiera implementar, [42]. 
6.Estudio de casos de éxito en Colombia - PCH La Chorrera de Puerto Rico.

\subsection{Localización del sitio de interés}

La cuenca de la quebrada La Chorrera de Puerto Rico se encuentra localizada en la subregión Oriente del Departamento de Antioquia en la zona occidental del municipio de San Carlos, Colombia, el cual limita por el norte con los municipios de San Rafael, San Roque y Caracolí, por el este con el municipio de Puerto Nare, por el sur con los municipios de Puerto Nare y San Luis y por el oeste con los municipios de Granada y Guatapé. Hasta el sitio de captación, la cuenca tiene un área aproximada de $4,3 \mathrm{~km}^{2},[43],[44]$.

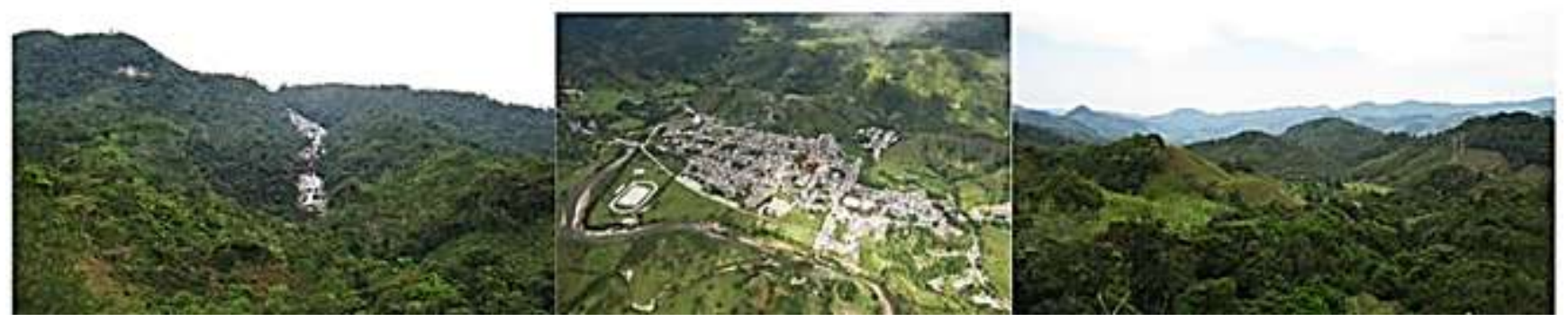

Figura 11. La Chorrera de Puerto Rico - Panorámica de San Carlos, Antioquia Arqueológico Quebrada La Chorrera [44].

\subsection{Características de la cuenca}

La cuenca de la quebrada La Chorrera de Puerto Rico se ha analizado considerando la cota de captación 1700 msnm y la cota de descarga 1280 msnm que son los sitios planteados para el aprovechamiento hidroeléctrico de esta corriente natural $[44]$.

\subsection{Estimación del recurso hídrico}

Se presenta de forma general la información de caudales utilizada para el dimensionamiento de las estructuras y los análisis de generación del proyecto. El desarrollo detallado de la hidrología se puede observar con mayor detalle en el "Estudio Hidrológico para el Aprovechamiento Hidroeléctrico de la quebrada La Chorrera de Puerto Rico, 2012" [43].

\begin{tabular}{|l|c|c|}
\hline \multicolumn{1}{|c|}{ PARÁMETRO } & UNIDADES & SITIO ESTUDIADO \\
\hline Área de la cuenca & $\mathrm{km}^{2}$ & 4.34 \\
\hline Caudal medio & $\mathrm{m}^{3}-s$ & 0.5 \\
\hline Perímetro de la cuenca & $m$ & 10.008 \\
\hline Longitud del cauce principal & $\mathrm{km}$ & 4.4 \\
\hline Cota superior de la cuenca & $\mathrm{msnm}$ & 2325 \\
\hline Cota superior del cauce & $m s n m$ & 2125 \\
\hline Cota en el punto de control & $\mathrm{msnm}$ & 1700 \\
\hline Pendiente promedio del cauce & $\mathrm{msnm}$ & 8.7 \\
\hline
\end{tabular}

Tabla 7. Características morfométricas de la cuenca del proyecto [43].

\begin{tabular}{|l|l|}
\hline TIPO DE CAUDAL & \multicolumn{1}{|c|}{ DEFINICIÓN } \\
\hline Caudal Medio & $\begin{array}{l}\text { Es un valor estimado, con base en las medidas de caudales } \\
\text { durante un periodo de tiempo en particular, que se presenta } \\
\text { frecuentemente (equivalente o superior) en una corriente de } \\
\text { agua }[45] . \\
\text { Bajo esta consideración, se puede hablar de caudal medio } \\
\text { diario, mensual y/o anual partiendo de las condiciones de } \\
\text { implementación del estudio realizado y su aplicación en un } \\
\text { proyecto particular [46]. }\end{array}$ \\
\hline
\end{tabular}




\begin{tabular}{|l|l|}
\hline TIPO DE CAUDAL & \multicolumn{1}{|c|}{ DEFINICIÓN } \\
\hline Caudal Ecológico & $\begin{array}{l}\text { Son los flujos de agua, el momento de su aplicación y la } \\
\text { calidad de las aguas precisos para mantener los ecosistemas } \\
\text { de agua dulce y de los estuarios, así como los medios de } \\
\text { subsistencia y bienestar de las personas que dependen de los } \\
\text { mismos [47]. } \\
\text { Según el Estudio Nacional del Agua, se dice que el caudal } \\
\text { ecológico debe ser, como mínimo, el 25\% del mínimo de los } \\
\text { caudales medios mensuales multianuales [48]. }\end{array}$ \\
\hline Caudal Máximo & $\begin{array}{l}\text { Los caudales máximos se definen como aquellos que no deben } \\
\text { ser superados durante la operación y gestión ordinaria de las } \\
\text { infraestructuras hidráulicas [49]. }\end{array}$ \\
\hline Caudal de Diseño & $\begin{array}{l}\text { Es el caudal con que se proyecta el diseño de la PCH, tal que se } \\
\text { pueda garantizar la obtención de la potencia de generación } \\
\text { estimada [13]. } \\
\text { También puede considerarse desde el diseño y construcción } \\
\text { de la estructura hidráulica, siendo el flujo máximo que puede } \\
\text { correr en ella sin generar ningun cambios o afectación } \\
\text { negativa sobre el sistema[50]. }\end{array}$ \\
\hline
\end{tabular}

Tabla 8. Tipos de caudales aplicables a proyectos de PCHs. Fuente: Elaboración propia.

\begin{tabular}{|l|c|}
\hline \multicolumn{1}{|c|}{ ÍTEM } & PCH QUEBRADA LA CHORRERA DE PUERTO RICO \\
\hline Caudal medio & 0.50 \\
\hline Caudal ecológico & 0.10 \\
\hline Caudal de diseño & 0.50 \\
\hline Salto bruto & 420 \\
\hline Salta neto & 397 \\
\hline Capacidad instalada & 1.66 \\
\hline Hf & 23 \\
\hline Tipo de turbina & Pelton \\
\hline
\end{tabular}

Tabla 9. Condiciones de trabajo del proyecto hidroeléctrico La Chorrera de Puerto Rico [43].

\section{Conclusiones}

Al realizar un primer acercamiento al desarrollo de un proyecto energético de tipo hidroeléctrico se pueden evidenciar los factores más críticos vinculados y las posibles maneras de mitigar cualquier efecto negativo a causa de su puesta en operación. De esta manera, los pasos previos a la implementación de una PCH deben ser llevados conforme a la normatividad del país donde se desarrollará buscando garantizar el éxito de cada fase y la inclusión de estas plantas dentro del sistema eléctrico interconectado nacional.

En este documento se presentaron dos esquemas de criterios básicos de implementación de PCHs que permiten observar el cómo cada fase o paso transforma el proyecto para que sea de provecho para una comunidad en particular. Así, la propuesta que se busca generar al finalizar este estudio estará orientada hacia un análisis exhaustivo y detallado de los distintos factores que se encuentran en cada modelo tal que, sea viable de realizar y disminuya los márgenes de falla de un proyecto energético en su fase de formulación y aprobación.

Al concluir esta etapa del estudio, se ha evidenciado la existencia de grandes potenciales energéticos alternativos que, por causas de desconocimiento o de inversión, no han sido explotados en pro del 
mejoramiento de la calidad de vida de muchas comunidades alejadas de las diferentes metrópolis de una nación. El lograr una estrategia de estudio de implementación de una Pequeña Central Hidroeléctrica, garantizará una disminución en los legalismos de algunos lugares o entidades gubernamentales y ayudará a hacer más viable la inversión en este tipo de proyectos que buscan llevar alternativas energéticas a zonas de difícil distribución por factores ajenos a los usuarios.

\section{Reconocimientos}

El trabajo presentado en este documento hace parte de una investigación desarrollada en el grupo de investigación Orden Y Caos - ORCA, del programa Ingeniería en Control de la Universidad Distrital Francisco José de Caldas. Los autores reconocen y expresan agradecimiento a la Universidad Distrital Francisco José de Caldas y al programa de Tecnología Electrónica, Ingeniería en Control e Ingeniería en Telecomunicaciones por el apoyo brindado durante la producción de este artículo y todos los procesos vinculados a él.

\section{Referencias}

[1] The Institute of Electrical and Electronics Engineers (IEEE), "IEEE Taxonomy Version $1.0 ", 2017$.

[2] Dirección general de Energía Comisión de comunidades Europeas, "Manual de Pequeña Hidráulica. Como llevar a bien fin un proyecto de minicentral hidroeléctrica”. Bruselas, Bélgica, 1998, pp. 282.

[3] CELSIA, "Filo de agua", 2017. [En línea]. D i s p o n i b l e e n : http://www.celsia.com/es/filo-de-agua

[4] E. Henao, "Pequeñas centrales hidroeléctricas", 2015 , pp. 386.

[5] M. Palacios Tejada, "Estudio de Prefactibilidad para la Construcción y Operación de la Pequeña Central Hidroeléctrica Río La Virgen en el Municipio de Masagu, Escuintla, Guatemala, 2007”, tesis M.Sc., Universidad de San Carlos de Guatemala, Guatemala, 2009.

[6] S. Morales, L. Corredor, J. Paba y L. Pacheco, "Etapas de desarrollo de un proyecto de pequeñas centrales hidroeléctricas: Contexto y criterios básicos de implementacion”, DYNA, vol. 81, no. 184, 2014, pp. 178-185.

[7] Empresa de Acueducto y Alcantarillado de Bogotá, "Pequeñas Centrales Hidroeléctricas en la EAAB", 2009.

[8] F. E. Sierra Vargas, A. F. Sierra Alarcon y C. A. Guerrero Fajardo, "Pequeñas y microcentrales hidroeléctricas: alternativa real de generación eléctrica”, Informador Técnico, vol. 75, 201 1, pp. 73-85.
[9] T. Gower, A. Rosenberger, A. Peatt y A. Hill, "Tamed Rivers: A Guide to River Diversion Hydropower in British Columbia", Watershed Watch Salmon Society, 2012, pp. 64.

[10] E. Briceño, R. Escobar y R. Saúl, Manual de capacitacion en operación y mantenimiento de pequeñas centrales hidraulicas". Lima: Soluciones Prácticas-ITDG, 2008.

[11] IDAE, "Manuales de Energias Renovables 6: Minicentrales Hidroeléctricas”, Madrid, 2006.

[12] Obechile, "Obechile construirá la Central Hidroeléctrica 'La Montaña 1'”, 2015. [En lín e a ]. D i s po n i ble e n : https://obechile.wordpress.com/2014/10/0 $1 /$ obechile-construira-la-centralhidroelectrica-la-montana-1/

[13] D. C. Navarro Mora y J. M. Liévano Hurtado, "Guía para estudios de prefactibilidad de pequeñas centrales hidroeléctricas como parte de sistemas híbridos", tesis, Universidad Javeriana, Bogotá, Colombia, 2004.

[14] Green For Growth Fund, "Financing hydropower Introduction to the Specifics of Small Hydro Power Plants", Frankfurt, Alemania, 2012.

[15] M. Balmer y D. Spreng, "Hydroelectric Power”. Amsterdam: Elsevier Ltd., 2008, pp. 193-209.

[16] A. Kunwor, "Technical Specifications of Micro Hydro Systems Design and its 
Implementation: Feasibility Analysis and Design of Lamaya Khola Micro Hydro Power Plant”, tesis, Arcada, 2012.

[17] J. Džaferović, "A Feasibility Study for a Small Hydropower Plant in B\&H”, tesis M. Sc., Universidad de Sarajevo, Sarajevo, 2012.

[18] O. Korkmaz, "A case study on feasibity assessment of small hydropower scheme", tesis M. Sc., Universidad Técnica de Medio Oriente, Turquía, 2007.

[19] International Centre on Small Hydro Power(ICSHO), "Feasibility Study Report of India Muerta Small Hydro Power Plant In Uruguay”, Uriguay, 2013.

[20] O. D. Lozano, "Oportunidades de inversión en Pequeñas Centrales Hidroeléctricas (PCH's)", 2015.

[21] Dirección de Licencias Permisos y Trámites ambientales, "Estudio de Impacto Ambiental - Construcción y operación de centrales hidroeléctricas generadoras", Colombia, 2006.

[22] Ministerio de Ambiente, "Estudio de Impacto Ambiental Proyecto Gramalote", Colombia, 2015.

[23] Ministerio de Ambiente Vivienda y Desarrollo Territorial, "Estudio de impacto ambiental - Proyectos de dragado de profundización de canales navegables y en áreas de deltas PU-TER-1-O3”, Colombia, 2006.

[24] Comisión de Regulación de Energía y Gas, "Reglamento de comercialización de energía eléctrica", Colombia, 2010.

[25] Servicio Transporte de Energía, "Estudio de conexión al sistema de transmisión nacional - S T N-del proyecto Hidroeléctrico Porce III - 685 MW”, Colombia, 2001.

[26] Asociación Colombiana de Ingenieros, "Estrategias Identificación De Riesgos Ambientales ( Eia - Daa ) En El
Desarrollo De Proyectos PCH's", Colombia, 2015.

[27] Celec, "Estudio de impacto ambiental definitivo (EIAD)”, Ecuador, 2013.

[28] Ministerio de Ambiente y Desarrollo Sostenible, "Manual para la Asignación de Compensaciones por Pérdida de Biodiversidad", Colombia, 2012.

[29] J. Cunha y P. V. Ferreira, "A Risk Analysis of Small-Hydro Power (SHP) Plants Investments", International Journal of Sustainable Energy Planning and Management, vol. 2, 2014, pp. 47-62.

[30] G. Bonthuys, M. van Dijk y J. Bhagwan, "A Feasibility and Implementation Model of Small-Scale Hydropower Development for Rural Electrification in South Africa: Design Chart Development", Water $S A$, vol. 42, no. 4, 2016.

[31] Renewables First, "Hydropower Turbine Types”, 2015. [En línea]. Disponible en: https://www.renewablesfirst.co.uk/hydr opower/hydropower-turbines/.

[32] Renewables First, "Archimedean Screw Hydro Turbine”, 2015. [En línea]. D i s p o n i b l e e n : https://www.renewablesfirst.co.uk/hydr o power / hydropower-learningcentre/archimedean-screw-hydroturbine/.

Renewables First, "Crossflow Turbines", 2015. [En línea]. Disponible en: https://www.renewablesfirst.co.uk/hydr opower / hydropower-learningcentre/crossflow-turbines/.

Renewables First, "Pelton and turgo turbines”, 2015. [En línea]. Disponible en: https://www.renewablesfirst.co.uk/hydr o power / hydropower-lear n ingcentre/pelton-and-turgo-turbines/.

[35] L. Stonecypher, "Hydraulic Turbines: Kaplan Turbine”, 2009. [En línea]. D i s p o n i ble e n : 
http://www.brighthubengineering.com/f luid-mechanics-hydraulics / $27426-$ hydraulic-turbines-kaplan-turbine/.

[36] K. Kuhl, K. Lai y D. Lainel, "Kaplan Turbine", 2014. [En línea]. Disponible en: https://www.engr.colostate.edu/ pierre /ce_old/classes/CIVE\%20401/Team\%2 $\begin{array}{llllllllllllll}\mathrm{O} & \mathrm{r} & \mathrm{e} & \mathrm{p} & \mathrm{o} & \mathrm{r} & \mathrm{t} & \mathrm{s} & / & 8 & \% & 2 & \mathrm{O} & -\end{array}$ \%20Kaplan\%20Kuhl\%20Lai\%20Lainel.p df.

[37] M.Harano, K. Tani y S. Nomoto, "Practical application of high-performance francisturbine runner fitted with splitter blades at ontake and shinkurobegawa no. 3 power stations of the Kansai electric power CO.", Hitachi Rev., vol. 55, no. 3, 2006, pp. 109-113.

[38] G. P. Heckelsmueller, "Application of variable speed operation on Francis turbines", Ing. e Investig., vol. 35, no. 1, 2015, pp. 12-16.

[39] E. J. Bustamante Cabrera y C. P. Arias Reyes, "Diseño y construcción de una turbina pelton para generación eléctrica, capacidad 2 kw.", Kimerius, 2008.

[40] VOITH Hydro, "Pelton turbines", 2013. [En línea]. Disponible en: http://voith.com/corp-en/turbinesgenerators / turbines/peltonturbines.html

[41] H. Ramos y A. B. de Almeida, "Small Hydropower Schemes as an Important Renewable Energy Source”, 2007.

[42] A. Nava Mastache y H. García Gutiérrez, "Selección y dimensionamiento de turbinas hidráulicas para centrales hidroeléctricas”, Universidad Nacional Autónoma de Mexico, 2013.

[43] LHC Consultores Ambientales, "Estudio de Impacto Ambiental PCH La Chorrera San Carlos, Antioquia”, Colombia, 2012.

[44] LHC Consultores Ambientales, "Proyecto
Hidroeléctrico La Chorrera de Puerto Rico San Carlos - Antioquia Diagnóstico y Evaluación del Impacto Arqueológico", Colombia, 2012.

[45] H. Gonzalo Rivera, E. Domínguez, R. Marin Ramírez $\mathrm{y}$ R. Vanegas, "Metodología de Cálculo del Índice de escasez de agua superficial”, Lima, Perú: INEI, 2004.

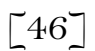

C. Palacios Santa-Cruz, "Caudales de diseño en el río Piura y su variación histórica ante el fenómeno El Niño”, tesis, Universidad de Piura, Piura, Perú, 2010.

[47] Servicio de Evaluación Ambiental, "Guía Metodológica para Determinar el Caudal Ambiental para Centrales Hidroeléctricas en el SEIA”, Chile: Servicio de Evaluación Ambiental, 2016.

[48] IDEAM, "Estudio Nacional del Agua", Bogotá, Colombia, 2015,

[49] A. M. Fernández Santamarina y M. Cebrián del Moral, "Conceptos y Métodos sobre el régimen de caudales ecológicos”, España: Solitario, 2011.

[50] Alternativas Viales, "Hidrología e hidráulica - Capítulo III - Tomo 1 - Tramo Ibagué - Honda”, Colombia, 2013 